\title{
A METHOD TO SOLVE MISSILE-AIRCRAFT PURSUIT-EVASION DIFFERENTIAL GAMES
}

\author{
Fumiaki Imado* and Takeshi Kuroda** \\ *Department of Mechanical Systems Engineering, Shinshu University \\ 4-17-1 Wakasato Nagano, Nagano 380-8553 JAPAN \\ **Advanced Technology Laboratory, Mitsubishi Electric Corporation \\ 8-1-1 Tsukaguchi Honmachi Amagasaki Hyogo 661-8661 JAPAN
}

\begin{abstract}
This paper shows; that, in some conditions we can obtain the exact solution for a missile and an aircraft differential games which minimizes and maximizes the essential pay-off of the problem: the miss distance, without employing any linearized approximation. The idea of our method is, by starting from the aircraft one-sided optimal control solution against a PNG missile, which maximizes the miss distance, and then optimize the missile control to minimize the miss distance. The obtained minimax solution shows the resultant miss distance is far smaller than that of the PNG missile. Although it is impossible to obtain the solution in real time, however, if the results are incorporated into the missile guidance system as a knowledge base, the performance will be fairly improved. The extension of the study into more general cases is also explained. Copyright $\odot 2005$ IFAC
\end{abstract}

Keywords: Missile Guidance, Differential Ga mes, Pursuit-Evasion, Missiles, Aircraft

\section{INTRODUCTION}

Many studies have appeared about pursuit-evasion dynamic games between a missile and an aircraft, however, none of them has obtained the exact solution which minimizes and maximizes the essential pay-off of the problem: the miss distance (MD). Many papers have obtained minimax-time solutions between two vehicles under linearized approximation, however, as far as the missile-aircraft pursuit-evasion game is concerned, there is little importance of minimizing and maximizing the interception time. If the exact solution which minimizes and maximizes the MD (Let us call this exact solution "ESMD") is obtained, and the characteristics of the ESMD are studied, we will be able to introduce the knowledge into a missile guidance system, and improve the performance. This paper shows an approach to obtain the ESMD for pursuit-evasion differential games between missile and aircraft without employing any linearized approximation. Our former studies (Imado and Miwa, 1983), (Imado and Miwa, 1986), (Imado and Uehara, 1998), (Imado, 2001) have shown that, the optimal aircraft maneuver against a proportional navigation guidance (PNG) missile becomes a horizontal-S type or a vertical-S type. As the PNG is a suboptimal control for an evasive target, the solution may be 
considered as an approximation of the precise differential game solution. The idea of this study is: to start from the aircraft a one-sided optimal evasive control solution against a PNG missile, which maximizes the MD, and then improving the missile control to minimize the MD. The minimax solution is iteratively corrected, and finally we could obtain the exact minimax solution for this problem. The detail of the problem, the algorithm for the solution and an example result is shown in this paper. As the initial geometry in this study is rather limited, the method of extending the study into more general cases is also described.

\section{MATHEMATICAL MODEL}

Fig. 1 shows the relative geometry of the pursuer and the evader and symbols. For the reason explained later, the motions are constrained in a vertical plane. In the paper, the pursuer is a missile, and the evader is an aircraft. The following equations of motion are used for calculation of the optimal controls for both vehicles. The aircraft is modeled as a point mass, and the equations of motion in a vertical plane are

\section{Aircraft Motion}

$$
\begin{aligned}
\dot{v}_{t} & =\left(T_{t} \cos \alpha-D\right) / m_{t}-g \sin \gamma_{t} \\
\dot{\gamma}_{t} & =\left(L+T_{t} \sin \alpha\right) /\left(m_{t} v_{t}\right) \\
& \quad-\left(g / v_{t}\right) \cos \gamma_{t} \\
\dot{x}_{t} & =v_{t} \cos \gamma_{t} \\
\dot{h}_{t} & =v_{t} \sin \gamma_{t}
\end{aligned}
$$

where

$$
\begin{aligned}
& L=(1 / 2) \rho v_{t}^{2} S_{t} C_{L} \\
& D=(1 / 2) \rho v_{t}^{2} S_{t} C_{D}
\end{aligned}
$$

where

$$
\begin{aligned}
& C_{L}=C_{L \alpha}\left(\alpha-\alpha_{0}\right) \\
& C_{D}=C_{D 0}+k C_{L}^{2}
\end{aligned}
$$

A constraint is imposed on the value of the aircraft lateral acceleration $a_{t}$, which is treated as the aircraft control variable.

$$
a_{t}=L / m_{t} \leq a_{t \max }
$$

\section{Missile Motion}

The missile lateral acceleration is approximated by a first-order lag to a lateral acceleration command.

$$
\begin{aligned}
& \dot{v}_{m}=1 / m_{m}\left(T_{m}-D_{m}\right) \\
& \dot{a}_{m}=\left(a_{m c}-a_{m}\right) / \tau \\
& \dot{\gamma}_{m}=a_{m} / v_{m} \\
& \dot{x}_{m}=v_{m} \cos \gamma_{m} \\
& \dot{h}_{m}=v_{m} \sin \gamma_{m}
\end{aligned}
$$

$$
\begin{aligned}
& D_{m}=k_{1} v_{m}{ }^{2}+k_{2}\left(a_{m} / v_{m}\right)^{2} \\
& k_{1}=(1 / 2) \rho_{m} S_{m} C_{D 0 m} \\
& k_{2}=2 k_{m} m_{m}{ }^{2} /\left(\rho_{m} S_{m}\right) \\
& T_{m}(t)=\left\{\begin{array}{lll}
T_{m} \text { for } 0<t \leq t e \\
0 & \text { for } t_{e}<t
\end{array}\right.
\end{aligned}
$$

The calculation method of optimal missile acceleration command $a_{m c}$, which is treated as the missile control variable is explained later. On the other hand, for the PNG missile with signal saturation taken into consideration, $a_{m c}$ is given by

$$
a_{m c}= \begin{cases}N_{e} v_{c} \dot{\sigma} & \text { for }\left|a_{m c}\right| \leq a_{m c \max } \\ a_{m c \max } \operatorname{sign}\left(a_{c}\right) \text { for } & \left|a_{m c}\right|>a_{m c \max }\end{cases}
$$

In Eq.(17), $N_{e}$ is the effective navigation ratio, $v_{c}$ the closing velocity, and $\sigma$ the line-of-sight turning rate given by

$$
\begin{aligned}
& v_{c}=-\dot{r} \\
& \dot{\sigma}=1 / r^{2}\left[\left(h_{t}-h_{m}\right)\left(\dot{x}_{t}-\dot{x}_{m}\right)\right. \\
& \left.\quad-\left(\dot{h}_{t}-\dot{h}_{m}\right)\left(x_{t}-x_{m}\right)\right]
\end{aligned}
$$

where $r$ is slant range,

$$
r=\left[\left(x_{t}-x_{m}\right)^{2}+\left(h_{t}-h_{m}\right)^{2}\right]^{1 / 2}
$$

\section{SOME FEATURES OF THE GAME SOLUTION}

Some features of the optimal evasive maneuvers of vehicles in a plane are well depicted in (Imado and Miwa, 1983), (Imado and Miwa, 1986), (Imado, 2001). Fig. 2 shows a typical trajectory and control histories of an aircraft against a PNG missile. In head on cases, if the initial relative distance is large enough, the evader at first takes the maximum lateral acceleration and inverse the direction, then accelerates longitudinally. The missile optimal control is easily obtained in this case, however, the solution has little importance. If the initial distance is small, the evader takes the maximum lateral acceleration to one side at first, then at an appropriate time, it changes the sign of its acceleration and takes

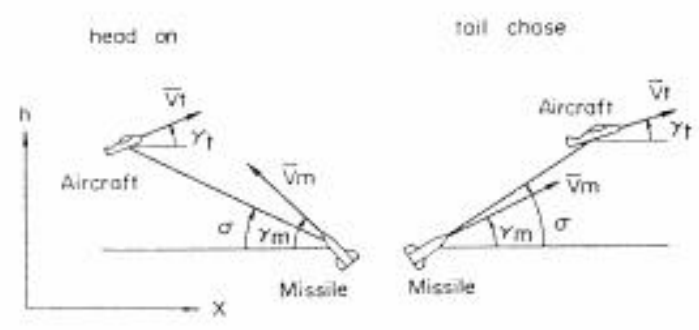

Fig.1 Geometry and symbols 
the maximum lateral acceleration to the opposite side. On the other hand, if the initial distance is between these two cases,. the optimal control of the evader will become as shown in Fig.2.

\section{EXACT SOLUTION OF THE GAME}

In this paper, a differential game solution is obtained in reference to the short range case. In the case of a horizontal plane, the symmetrical optimal maneuver exists, however, in the case of a vertical plane, the optimal aircraft maneuver against a PNG missile is, first to take the maximum $g$ upward, and at an appropriate time, inverse the aircraft attitude and take the maximum $g$ downward. The maneuver is called a "split-S". To understand why the downward split-S produces a larger MD than the symmetrical maneuver (first downward, next upward), refer to (Imado and Miwa, 1983). However, as the missile does not know whether the aircraft will continue the maximum upward $\mathrm{g}$, or reverse the direction at any time, therefore the optimal missile control should be the one that minimizes the MD in both cases. As the result, the missile takes at first a smaller upward g, and follows the aircraft to cope with both aircraft maneuvers. The missile and aircraft initial relative geometry is shown in Fig.1, where appropriate altitude and distance are set. (see Table 1) The steps to obtain this differential game solution are as follows.

Step 1. Obtaining nominal trajectories and controls by PNG

(1)Provide a set of the aircraft maneuvers. The aircraft takes the maximum $g$ upward, and inverses its attitude at an arbitrary time, and takes the maximum $g$ downward. The arbitrary times are set e.g. with $0.1 \mathrm{~s}$ interval from the initial time until interception, such as $0 \mathrm{~s}, 0.1 \mathrm{~s}, 0.2 \mathrm{~s}, \ldots . .5 .9 \mathrm{~s}, 6.0 \mathrm{~s}$. Let us call this time set $\{0 \mathrm{~s}, 0.1 \mathrm{~s}, 0.2 \mathrm{~s}, \ldots, 5.9 \mathrm{~s}$, $6.0 \mathrm{~s}\}=\left\{t_{1}, t_{2}, \ldots, t_{n}-1, t_{n}\right\}$ as $\left\{t_{i}\right\}$ and corresponding maneuvers set as "maneuver $\left\{A_{i}\right\}$ ". The last component of the $\left\{A_{i}\right\}$ is the maneuver, which takes the maximum $\mathrm{g}$ upward throughout until interception. Let us call this maneuver "maneuver $B$ ".

(2)The missile is guided by PNG with the effective navigation gain $N_{e}$ is set to be $N_{e \max }=4.0$, and simulations are conducted against maneuvers $B$ and $A_{i}$, where $i$ is increased one by one. For small “ $i$ "s, terminal miss distances (MDs) are $0 \mathrm{~m}$ against both $B$ and $A_{i}$, but for a value of a larger " $i$ ", a small $\mathrm{MD}$ is produced against the maneuver $A_{i}$.

(3)For this case, the value of the $N_{e}$ at $0 \leq t<t_{i}$
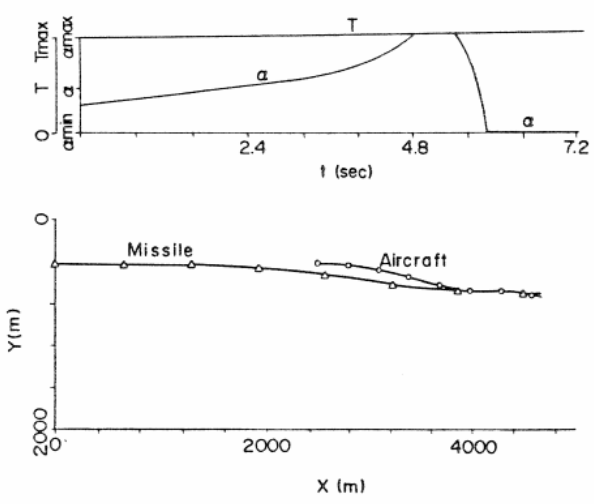

Fig.2 Optimal aircraft evasive maneuver against a PNG missile (tail chase case)
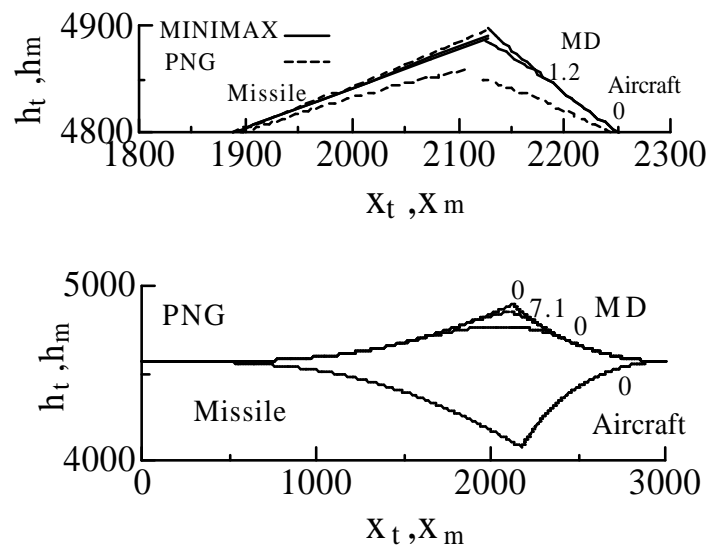

Fig.3 An exact solution of missile-aircraft pursuit-evasion problems

Table 1 Parameters

\begin{tabular}{l}
\hline Aircraft (afterburner) \\
$m_{t}=7500 \mathrm{~kg}$ \\
$h_{t 0}=4572 \mathrm{~m}$ \\
$S_{t}=26 \mathrm{~m}^{2}$ \\
$v_{t 0}=290.2 \mathrm{~m} / \mathrm{s} \quad(0.9 \mathrm{M})$ \\
$C_{L \alpha}=3.689 / \mathrm{rad}$ \\
$C_{D 0}=0.0224 \quad k=0.260$ \\
$a_{t \max }=9 \mathrm{~g}$ \\
Engine $:$ PWF-100 $T_{\max }=T_{\max }(v, h)$ \\
Missile $($ Sustainer phase $)$ \\
$m_{m 0}=176 \mathrm{~kg} \mathrm{ISP}=250 \mathrm{~s}$ \\
$T_{m}=6000 \mathrm{~N}$ \\
$t_{e}=8 \mathrm{~s}$ \\
$S_{m}=0.0324 \mathrm{~m}^{2}$ \\
$h_{m 0}=4572 \mathrm{~m}$ \\
$v_{m 0}=644.6 \mathrm{~m} / \mathrm{s} \quad(2.0 \mathrm{M})$ \\
$C_{L \alpha}=35.0 / \mathrm{rad}$ \\
$C_{D 0}=0.90 \quad k=0.030$ \\
$a_{c \max }=40 \mathrm{~g}$ \\
$r_{0}=3000 \mathrm{~m}(\mathrm{head}$ on $)$ \\
\end{tabular}


is decreased a small value, until the MD against $A_{i}$ is diminished, while against $B$ is still $0 \mathrm{~m}$. Note that, the $N_{e}$ at $t_{i} \leq t$ is still 4.0. Next, $i$ is increased and if a MD is produced, the same process is taken. Finally, we can find the " $i$ ", where against both maneuvers $B$ and $A_{i}$, some MDs (both are not insignificant) are produced. Then we adjust the value of the $N_{e}$ at $0 \leq t<t_{i}$ by increasing or decreasing so that both $\mathrm{MD}$ against $B$ and $A_{i}$ become equal. Let us denote the corresponding value of the $N_{e}$ and $t_{i}$, as $N_{e} 0$ and $t_{p}$ respectively. We will call the point "Non zero miss point"

(4)We employ the PNG with the value of

$$
\begin{array}{lll}
N_{e}=N_{e 0} & \text { for } & 0 \leq t<t_{p} \\
N_{e}=N_{e \max } & \text { for } & t_{p} \leq t
\end{array}
$$

The process is continued by increasing the value $i$ one by one, throughout all sets of $\left\{t_{i}\right\}$. The value of MD against $B$ and $A_{i}$ increases at first, reaches to a maximum value, then decreases, and finally it becomes 0 . Let us call the obtained set of $a_{m c}(t)$ by PNG as $\left\{a^{*}{ }_{m c}(t)\right\}$. Above processes are easily worked out, because all calculations are conducted only by simulations which do not include any optimizing process.

Step 2. Optimizing the missile control

(1)The obtained set in step 1 is employed as nominal trajectories and controls in order to obtain optimal controls of $a^{0}{ }_{m c}(t)$ which minimize the MD. The optimizing algorithm is the steepest ascent method (Bryson Jr, A.E. and Denham, W.F., 1962) shown in Appendix. In the optimization process, the initial missile condition is given by the states at $t_{p}$ obtained in step 1, and optimal controls until interception are calculated against the aircraft maneuvers $B$ and $A_{i}$.

(2)As the optimal controls are more effective than PNG controls, MDs will be 0 against both $B$ and $A_{i}$, however for a larger time $t_{i}$, some MDs will be produced. Suppose that at a time $t_{i}$, the missile altitude is $h_{m i}$, and optimal controls between $t_{i} \leq t \leq t_{f}$ against maneuvers $B$ and $A_{i}$ produced different values of MDs, then the missile altitude at $t_{i} ; h_{m i}$ is increased or decreased in order to produce the same MD value against $B$ and $A_{i}$, in the same way as we have adjusted $a_{m c}(t)$ in step 1. Let us denote the obtained $h_{m i}$ as $h^{*} m i$.

(3)With the concept of energy maneuverability, it is natural that a larger $v_{m}$ produces a smaller MD. Therefore, we calculate the optimal $a_{m c}(t)$ which maximizes $v_{m}\left(t_{i}\right)$ between $0 \leq t \leq t_{i}$, where $h_{m}\left(t_{i}\right)=h_{m i}^{*}$ is constrained.
(4)The change of $a_{m c}(t)$ in (3) causes the change of missile state at $t_{i}$.Therefore (2) and (3) are repeated and through this iteration process, optimal missile state at $t_{i}$ and optimal control $a_{m c}^{0}(t), \quad\left(0 \leq t \leq t_{i}\right)$ as well as $a_{m c}^{0}(t)$ $\left(t_{i}<t \leq t_{f}\right)$ are obtained.

(5)Work out this process by increasing the value $i$ of $\left\{t_{i}\right\}$ one by one, throughout all components of $\left\{t_{i}\right\}$. Figure 3 shows an example of the results. In the upper figure, PNG missile vs optimal aircraft trajectories as well as the MDs are shown where the maximum MD is $7.1 \mathrm{~m}$. On the other hand, in the lower figure, solid lines show the missile and aircraft minimax trajectories. The minimax pay-off in this case is $1.2 \mathrm{~m}$, which shows a great reduction of the MD by employing the missile guidance based on the game solution. Table 1 shows some parameters employed.

Necessary conditions of the solution

Although the possibility of the local solution of this result can not be denied, necessary conditions of the solution are numerically verified as follows.

$$
\begin{aligned}
& \frac{\partial H}{\partial a_{m c}}=0 \quad \text { or } \quad\left|a_{m c}\right|=a_{c \max } \\
& \text { for } t_{s} \leq t \leq t_{f} \\
& \frac{\partial H}{\partial a_{t}}=0 \quad \text { or } \quad\left|a_{t}\right|=a_{t \max } \\
& \text { for } 0 \leq t \leq t_{f}
\end{aligned}
$$

where $t_{s}$ is the time when the minimax trajectory of the vehicles branches into two curves, and the system Hamiltonian function $H$ is defined by

$$
\begin{aligned}
& H=\left(\frac{\partial \bar{f}}{\partial \bar{u}}\right)^{T}\left(\bar{\lambda}_{\phi \Omega}-\lambda_{\psi \Omega} \bar{v}\right) \\
& \bar{u}=\left(a_{m c}, a_{t}\right)
\end{aligned}
$$

As for symbols employed in above equations, see the appendix. The above characteristics are automatically satisfied by the steepest ascent algorithm. The saddle point condition is satisfied in the result.

$$
\begin{gathered}
J\left[a_{m c}^{0}(t), a_{t}(t)\right]<J\left[a_{m c}^{0}(t), a_{t}^{0}(t)\right]<J\left[a_{m c}(t), a_{t}^{0}(t)\right] \\
\text { for } 0 \leq t \leq t_{f}
\end{gathered}
$$

where $a_{m c}^{0}(t)$ and $a_{t}^{0}(t)$ are optimal solutions of $a_{m c}(t)$ and $a_{t}(t)$, respectively. The above characteristics are assured by the proposed algorithm. It is also verified by selecting arbitrary differential changes from the optimal control of one vehicle and calculating corresponding one-sided optimal control 
of the opponent vehicle and conducting simulations.

\section{THE EXTENSION OF THE STUDY}

The example result obtained in the previous section may not be so impressive. Another type of optimal aircraft evasive maneuver against PNG missile shown in Fig. 2 will be more interesting. In the case, the initial geometry is tail chase, and the aircraft employs its angle-of-attack $\alpha$ as the control variable instead of $a_{t}$, but the controls $\alpha$ and $a_{t}$ are equivalent. The noticeable feature of this maneuver is that it employs an intermediate value of $\alpha$ in earlier time. The reason is considered as that, the aircraft drag is increased in proportion to $\alpha^{2}$ (or $a_{t}{ }^{2}$ ), the use of maximum $\alpha$ may not be optimal. Depending on the characteristics of the PWF-100 engine employed in this paper, the aircraft can avail the larger thrust at higher velocity and lower altitude. Therefore, it is expected that the aircraft initially takes an intermediate upward acceleration, and at a suitable time, dives downward with its maximum $a_{t}$. A study to find such a kind of minimax solution is currently being conducted. The algorithm is stated as follows.

Steps for a medium range case.

1.Define the time set $\left\{t_{i}\right\}$ like as in Sec.4. For each $t_{i},(i=1 \sim n)$, calculate the set of optimal controls of the aircraft and the missile which maximize the aircraft and missile velocities at $t_{i}$, respectively.

2.The above process produces the sets of terminal surfaces $\left\{\psi_{m, i}\right\}(i=1 \sim n)$ for the missile, and $\left\{\psi_{a, i}\right\}(i=1 \sim n)$ for the aircraft, respectively. From the arbitrary point $A_{i}$ on $\psi_{a, i}$, the aircraft takes maximum upward or downward accelerations. The trajectories of the aircraft are shown as $S_{a 1}$ and $S_{a 2}$ in Fig.4, respectively. Calculate the missile optimal controls which start from $\psi_{m, i}$, and minimize MD against $S_{a 1}$ and $S_{a 2}$. The following process are almost the same as that of sec.4, however, the calculation must be conducted through all terminal surfaces $\psi_{a, i}$. As the total process is severely time intensive, an efficient computer program is under development. The conceptual minimax trajectories of two vehicles are shown in Fig.5.

\section{CONCLUSION}

An exact solution for missile-aircraft pursuit-evasion games is obtained and the result is shown.

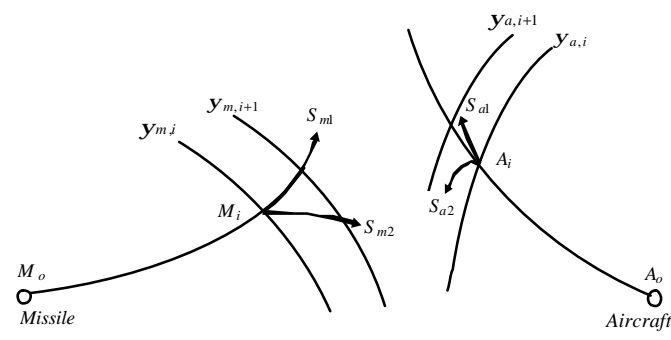

Fig.4 The calculation of the minimax solution for a medium range case

$\psi_{a, i}$ : A ircraft terminal (velocity maximum) surface at $t_{i}$

$\psi_{m, i}$ : Missile terminal (velocity maximum) surface at $t_{i}$

$S_{a 1}$ : Aircraft takes maximum upward acceleration at $A_{i}$

$S_{a 2}$ : Aircraft takes maximum downward acceleration at $A_{i}$

$S_{m 1}$ :Missile takes optimal control to minimize terminal miss against $S_{a 1}$ at $M_{i}$

$S_{m 2}$ : Missile takes optimal control to minimize terminal miss against $S_{a 2}$ at $M_{i}$

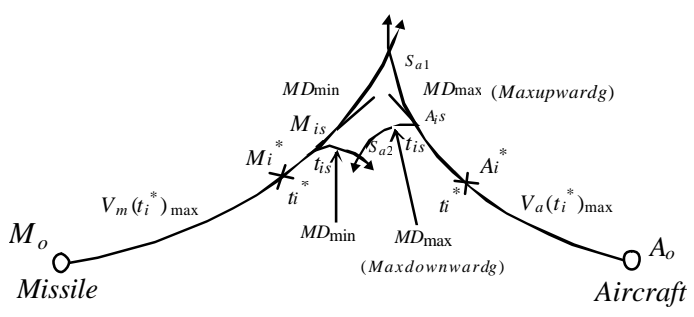

Fig.5 The concept of a minimax solution

The algorithm to solve this problem is explained in detail. The method of the extension of the study into more general cases is also explained.

\section{REFERENCES}

Imado, F. and Miwa, S.(1983) Optimal Evasive

Maneuver of a Fighter Against Proportional Navigation Missile, AIAA paper 83-2139, AIAA Atmospheic Flight Mechanics Conference.

Imado, F. and Miwa, S. (1986) Fighter Evasive Maneuvers Against Proportional Navigation Missile, Journal of Aircraft, Vol.23,No.11, pp.825-830.

Imado, F. and Uehara, S. (1998) High-g Barrel Roll Maneuvers Against Proportional Navigation from Optimal Control of View, Journal of Guidance, Control, and Dynamics, Vol.21, No.1, pp. 876-881.

Imado, F. (2001) The Features of Optimal Avoidance in Two-Dimensional Pursuit-Evasion Dynamic 
Games, Information Technology and Management, Vol.1, 1-8.

Bryson Jr, A.E. and Denham, W. F. (1962) A Steepest Ascent Method for Solving Optimum Programming Problems, Journal of Applied Mechanics, Vol.29, pp.247-257.

\section{Appendix}

A steepest ascent method (Bryson Jr. A.E. and Denham, W.F., 1962) is a well-known algorithm to solve nonlinear optimal control problems. A summary of the algorithm is shown here for the readers' convenience.

Find $\bar{u}(t)$ to maximize (for minimizing, change the sign of the following $\phi$ )

$$
J=\phi\left[\bar{x}\left(t_{f}\right)\right]
$$

where

$$
\begin{aligned}
& \dot{\bar{x}}=\bar{f}(\bar{x}, \bar{u}, t) \\
& \bar{x}\left(t_{0}\right)=\bar{x}_{0}: \text { specified }
\end{aligned}
$$

with terminal constraints

$$
\bar{\psi}\left[\bar{x}\left(t_{f}\right), t_{f}\right]=0
$$

where $\bar{x}(t)$ is an $n$-dimensional state vector, $\bar{u}(t)$ is an $m$-dimensional control vector, and $\bar{\psi}$ is a $q$-dimensional constraint vector. The terminal time $t_{f}$ is determined from the following stopping condition:

$$
\Omega\left[\bar{x}\left(t_{f}\right), t_{f}\right]=0
$$

The optimal control $\bar{u}(t)$ is obtained by the following algorithm.

1)Estimate a set of control variable histories $\bar{u}(t)$ (which is called a nominal control)

2)Integrate the system equations (A-2) with the initial condition (A-3) and control variable histories from step 1 until (A-5) is satisfied. Record $\bar{x}(t), \bar{u}(t)$, and $\bar{\psi}\left[\bar{x}\left(t_{f}\right)\right]$. Calculate the time histories of the $(n \times n)$ and $(n \times m)$ matrices of functions:

$$
F(t)=\frac{\partial \bar{f}}{\partial \bar{x}} \quad G(t)=\frac{\partial \bar{f}}{\partial \bar{u}}
$$

3)Determine $n$-vector influence functions $\bar{\lambda}_{\phi}(t)$, $\bar{\lambda}_{\Omega}(t)$ and $(n \times q)$ matrix of influence functions $\lambda_{\psi}(t)$, by backward integration of the following influence equations, using $\bar{x}\left(t_{f}\right)$ obtained in Step 2 to determine the boundary conditions:

$$
{\dot{\overline{\lambda_{\phi}}}}^{T}=-\overline{\lambda_{\phi}} \frac{\partial \bar{f}}{\partial \bar{x}}(\mathrm{~A}-7){\overline{\lambda_{\phi}}}^{T}\left(t_{f}\right)=\left(\frac{\partial \phi}{\partial \bar{x}}\right)_{t f}
$$

$$
\begin{aligned}
& \dot{\bar{\lambda}}_{\Omega}^{T}=-\bar{\lambda}_{\Omega} \frac{\partial \bar{f}}{\partial \bar{x}}(\mathrm{~A}-9) \bar{\lambda}_{\Omega}{ }^{T}\left(t_{f}\right)=\left(\frac{\partial \Omega}{\partial \bar{x}}\right)_{t f} \\
& \dot{\lambda}_{\psi}^{T}=-\lambda_{\psi}{ }^{T} \frac{\partial \bar{f}}{\partial \bar{x}}(\mathrm{~A}-1) \lambda_{\psi}{ }^{T}\left(t_{f}\right)=\left(\frac{\partial \bar{\psi}}{\partial \bar{x}}\right)_{t f}
\end{aligned}
$$

Calculate the following influence functions

$$
\begin{aligned}
& \bar{\lambda}_{\phi \Omega}=\bar{\lambda}_{\phi}-\frac{\dot{\phi}\left(t_{f}\right)}{\dot{\Omega}\left(t_{f}\right)} \bar{\lambda}_{\Omega} \\
& \lambda_{\psi \Omega}=\lambda_{\psi}-\frac{\dot{\bar{\psi}}\left(t_{f}\right)}{\dot{\Omega}\left(t_{f}\right)} \lambda_{\psi}
\end{aligned}
$$

4)Simultaneously with Step 3 ,compute the following integrals:

$$
\begin{aligned}
& I_{\psi \psi}=\int_{\psi}^{t f} \lambda^{T}{ }_{\psi \Omega} G W^{-1} G^{T} \lambda_{\psi \Omega} d t \\
& \bar{I}_{\psi \phi}=\int_{\psi t \phi f}^{t_{t(j)}} \lambda^{T}{ }_{\psi \Omega} G W^{-1} G^{T} \bar{\lambda}_{\phi \Omega} d t \\
& I_{\phi \phi}=\int_{t 0}^{T} \bar{\lambda}_{\phi \Omega} G W^{-1} G^{T} \bar{\lambda}_{\phi \Omega} d t
\end{aligned}
$$

5)Choose values of $\delta \bar{\psi}$ to cause the next solution to be closer to the desired values $\bar{\psi}\left[\bar{x}\left(t_{f}\right)\right]=0$. For example, one might choose

$$
\delta \bar{\psi}=-\varepsilon \bar{\psi}\left[\bar{x}\left(t_{f}\right)\right], \quad 0<\varepsilon \leq 1
$$

The proper choice of $\delta \bar{u}(t)$, which increase the $J$ is given as follows:

$$
\delta \bar{u}(t)=(1 / 2 \mu) W^{-1} G^{T}\left(\bar{\lambda}_{\phi \Omega}-\lambda_{\psi \Omega} \bar{v}\right)
$$

where

$2 \mu=\left[\frac{I_{\phi \phi}-\bar{I}_{\psi \phi}{ }^{T} I_{\psi \psi}{ }^{-1} \bar{I}_{\psi \phi}}{(d p)^{2}-d \bar{\psi}^{T} I_{\psi \psi}{ }^{-1} d \bar{\psi}}\right]^{\frac{1}{2}}$

$\bar{v}=-2 \mu I_{\psi \psi}{ }^{-1} d \bar{\psi}+I_{\psi \psi}{ }^{-1} \bar{I}_{\psi \phi}$

where $d p$ and $(m \times m)$ matrix of weighting functions $W(t)$ are chosen to satisfy

$(d p)^{2}=\int_{t 0}^{t f} \delta \bar{u}^{-T}(t) W(t) \delta \bar{u}(t) d t$

6)Repeat Steps 1-5, using an improved estimate of $\bar{u}(t)$

where

$$
\bar{u}(t)=\bar{u}(t)_{\text {old }}+\delta \bar{u}(t)
$$

The key technique of the algorithm is the proper choice of the value $d p$, which must be changed every step, and how to avoid dropping into local optima, and to reach to the global optimum. 